Supporting Information

\title{
Chemical modification of hydroxyl-terminated polybutadiene and its application in composite propellants
}

Pingan Zhang ${ }^{1}$, Wenjiayi Tan ${ }^{3}$, Xilong Zhang ${ }^{4 *}$, Juan Chen ${ }^{1}$, Jianmin Yuan ${ }^{2}$, Jianru

$\operatorname{Deng}^{1 *}$

1. College of Chemistry and Chemical Engineering, Hunan University, Changsha 410082, PR China

2. College of Material Science and Engineering, Hunan University, Changsha 410082, PR China

3. College of Chemical Engineering and Materials Science, Tianjin University of Science and Technology, Tianjin 300222, PR China

4. Hu Bei Sanjiang Aerospace Jianghe Chemical Technology Co., Ltd. Yichang 444200, PR China

Corresponding author: * E-mail: zhangxilong@hnu.edu.cn (Xilong Zhang)

E-mail: dengjianru@hnu.edu.cn (Jianru Deng) 


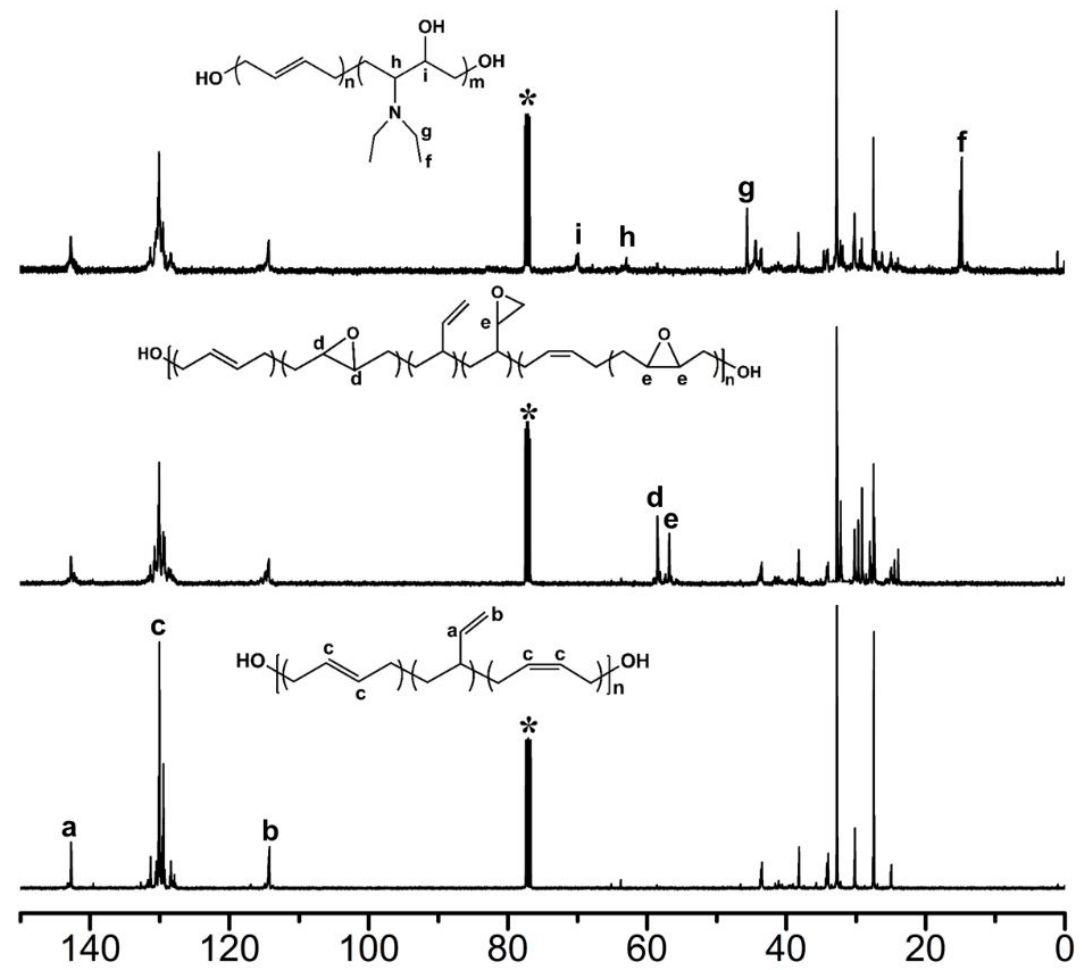

Figure S1 ${ }^{13}$ C-NMR spectra of (a)HTPB, (b)EHTPB, and (c)AEHTPB.

Table S1 Assignment of Chemical Shift $(\delta)$ from ${ }^{1} \mathrm{H}$ NMR of HTPB, EHTPB and AEHTPB

\begin{tabular}{|c|c|c|c|}
\hline$\delta, \mathrm{ppm}$ & & Hydrogen & \\
\hline & НTPB & ЕНТРВ & АЕНТРВ \\
\hline $5.7-5.1$ & $\begin{array}{l}-\mathrm{CH}=(1,2)+- \\
\mathrm{CH}=\mathrm{CH}-(1,4)\end{array}$ & $\begin{array}{c}-\mathrm{CH}=(1,2)+-\mathrm{CH}=\mathrm{CH}- \\
(1,4)\end{array}$ & $\begin{array}{c}-\mathrm{CH}=(1,2)+-\mathrm{CH}=\mathrm{CH}- \\
(1,4)\end{array}$ \\
\hline 5.4 & $-\mathrm{CH}=\mathrm{CH}-(1,4)$ & $-\mathrm{CH}=\mathrm{CH}-(1,4)$ & $-\mathrm{CH}=\mathrm{CH}-(1,4)$ \\
\hline $5.2-4.8$ & $=\mathrm{CH}_{2}(1,2)$ & $=\mathrm{CH}_{2}(1,2)$ & $=\mathrm{CH}_{2}(1,2)$ \\
\hline 4.1 & $-\mathrm{CH}_{2}-\mathrm{OH}\left(\mathrm{G}_{\mathrm{OH}}\right)$ & $-\mathrm{CH}_{2}-\mathrm{OH}\left(\mathrm{G}_{\mathrm{OH}}\right)$ & $-\mathrm{CH}_{2}-\mathrm{OH}\left(\mathrm{G}_{\mathrm{OH}}\right)$ \\
\hline $4.0-3.7$ & $-\mathrm{CH}_{2}-\mathrm{OH}\left(\mathrm{H}_{\mathrm{OH}}\right)$ & $-\mathrm{CH}_{2}-\mathrm{OH}\left(\mathrm{H}_{\mathrm{OH}}\right)$ & $-\mathrm{CH}_{2}-\mathrm{OH}\left(\mathrm{H}_{\mathrm{OH}}\right)$ \\
\hline $3.7-3.4$ & $-\mathrm{CH}_{2}-\mathrm{OH}\left(\mathrm{V}_{\mathrm{OH}}\right)$ & $-\mathrm{CH}_{2}-\mathrm{OH}\left(\mathrm{V}_{\mathrm{OH}}\right)$ & $-\mathrm{CH}_{2}-\mathrm{OH}\left(\mathrm{V}_{\mathrm{OH}}\right)$ \\
\hline $\begin{array}{c}3.55- \\
3.3\end{array}$ & & & $-\stackrel{\mathrm{C}}{\mathrm{C}} \mathrm{H}-\mathrm{OH}$ \\
\hline $\begin{array}{c}3.25- \\
3.1\end{array}$ & & & $-\stackrel{!}{\mathrm{C}} \mathrm{H}-\mathrm{N}$ \\
\hline $3.0-2.8$ & & -CH-CHO- $(1,4-c i s)$ & \\
\hline $2.8-2.4$ & & & $-\mathrm{CH}_{2-}$ (diethylamine) \\
\hline $2.8-2.6$ & & -CH-CHO- (1,4-trans) & \\
\hline $2.4-1.7$ & $\begin{array}{c}-\mathrm{CH}_{2}-(1,4)+-\mathrm{CH}- \\
(1,2)\end{array}$ & $\begin{array}{c}-\mathrm{CH}_{2}-(1,4)+-\mathrm{CH}- \\
(1,2)\end{array}$ & $\begin{array}{c}-\mathrm{CH}_{2}-(1,4)+-\mathrm{CH}- \\
(1,2)\end{array}$ \\
\hline $1.7-1.2$ & $-\mathrm{CH}_{2}-(1,2)$ & $-\mathrm{CH}_{2}-(1,2)$ & $-\mathrm{CH}_{2}-(1,2)$ \\
\hline
\end{tabular}


Table S2 Assignment of Chemical Shift $(\delta)$ from ${ }^{13} \mathrm{C}$ NMR of HTPB, EHTPB and AEHTPB

\begin{tabular}{|c|c|c|c|}
\hline \multirow[t]{2}{*}{$\delta, \mathrm{ppm}$} & \multicolumn{3}{|c|}{ Carbon } \\
\hline & HTPB & ЕНTPB & AEHTPB \\
\hline 142.6 & $\begin{array}{l}-\mathrm{CH}=(1,2)+- \\
\mathrm{CH}=\mathrm{CH}-(1,4)\end{array}$ & $\begin{array}{c}-\mathrm{CH}=(1,2)+-\mathrm{CH}=\mathrm{CH}- \\
(1,4)\end{array}$ & $\begin{array}{c}-\mathrm{CH}=(1,2)+-\mathrm{CH}=\mathrm{CH}- \\
(1,4)\end{array}$ \\
\hline $\begin{array}{c}132- \\
127\end{array}$ & $-\mathrm{CH}=\mathrm{CH}-(1,4)$ & $-\mathrm{CH}=\mathrm{CH}-(1,4)$ & $-\mathrm{CH}=\mathrm{CH}-(1,4)$ \\
\hline 114 & $=\mathrm{CH}_{2}(1,2)$ & $=\mathrm{CH}_{2}(1,2)$ & $=\mathrm{CH}_{2}(1,2)$ \\
\hline 70 & & & $-\mathrm{C} \mathrm{CH}-\mathrm{OH}$ \\
\hline 65.2 & $-\mathrm{CH}_{2}-\mathrm{OH}\left(\mathrm{G}_{\mathrm{OH}}\right)$ & $-\mathrm{CH}_{2}-\mathrm{OH}\left(\mathrm{G}_{\mathrm{OH}}\right)$ & $-\mathrm{CH}_{2}-\mathrm{OH}\left(\mathrm{G}_{\mathrm{OH}}\right)$ \\
\hline 63.6 & $-\mathrm{CH}_{2}-\mathrm{OH}\left(\mathrm{H}_{\mathrm{OH}}\right)$ & $-\mathrm{CH}_{2}-\mathrm{OH}\left(\mathrm{H}_{\mathrm{OH}}\right)$ & $-\mathrm{CH}_{2}-\mathrm{OH}\left(\mathrm{H}_{\mathrm{OH}}\right)$ \\
\hline 62.8 & & & $-\stackrel{I}{\mathrm{C}} \mathrm{H}-\mathrm{N}$ \\
\hline 58.5 & $-\mathrm{CH}_{2}-\mathrm{OH}\left(\mathrm{V}_{\mathrm{OH}}\right)$ & $-\mathrm{CH}_{2}-\mathrm{OH}\left(\mathrm{V}_{\mathrm{OH}}\right)$ & $-\mathrm{CH}_{2}-\mathrm{OH}\left(\mathrm{V}_{\mathrm{OH}}\right)$ \\
\hline 58.3 & & -CH-CHO- (1,4-trans) & \\
\hline 56.5 & & -CH-CHO- (1,4-cis) & \\
\hline 45.5 & & & $-\mathrm{CH}_{2-}$ (diethylamine) \\
\hline $47-44$ & $-\mathrm{CH}-(1,2)$ & $-\mathrm{CH}-(1,2)$ & $-\mathrm{CH}-(1,2)$ \\
\hline $39-35$ & $-\mathrm{CH}_{2}-(1,2)$ & $-\mathrm{CH}_{2}-(1,2)$ & $-\mathrm{CH}_{2}-(1,2)$ \\
\hline $33-31$ & $-\mathrm{CH}_{2}-(1,4-\operatorname{trans})$ & $-\mathrm{CH}_{2}-(1,4-\operatorname{trans})$ & $-\mathrm{CH}_{2}-(1,4-\operatorname{trans})$ \\
\hline $28-35$ & $-\mathrm{CH}_{2^{-}}(1,4-\mathrm{cis})$ & $-\mathrm{CH}_{2}-(1,4-\mathrm{cis})$ & $-\mathrm{CH}_{2}-(1,4-\mathrm{cis})$ \\
\hline 15 & & & $-\mathrm{CH}_{3}$ (diethylamine) \\
\hline
\end{tabular}

Table S3 Effect of reaction time on double bond conversion rate

\begin{tabular}{ccccc}
\hline Sample & EHTPB $_{10 \%}$ & EHTPB $_{15 \%}$ & EHTPB20\% & EHTPB $_{25 \%}$ \\
\hline Time (h) & 0.5 & 1.5 & 3 & 4 \\
\hline
\end{tabular}

a: Reaction conditions: HTPB $30 \mathrm{~g}$, dichloromethane $100 \mathrm{~mL}$, acetic acid $24 \mathrm{~g}$, sodium tungstate dihydrate $0.3 \mathrm{~g}, \mathrm{H}_{2} \mathrm{O}_{2}(30 \mathrm{wt} . \%) 60 \mathrm{~g}$, reaction temperature $55^{\circ} \mathrm{C}$

The double bond conversion can be calculated as follows:

$$
\mathrm{X}=\frac{\mathrm{A}_{2.7}+\mathrm{A}_{2.9}+0.5 \times \mathrm{A}_{2.5}}{\mathrm{~A}_{2.7}+\mathrm{A}_{2.9}+0.5 \times \mathrm{A}_{2.5}+\mathrm{A}_{5.4}+0.5 \mathrm{~A}_{4.9}} \times 100 \%
$$

where $A_{i}$ is the area of the peak at $i \mathrm{ppm}$. 

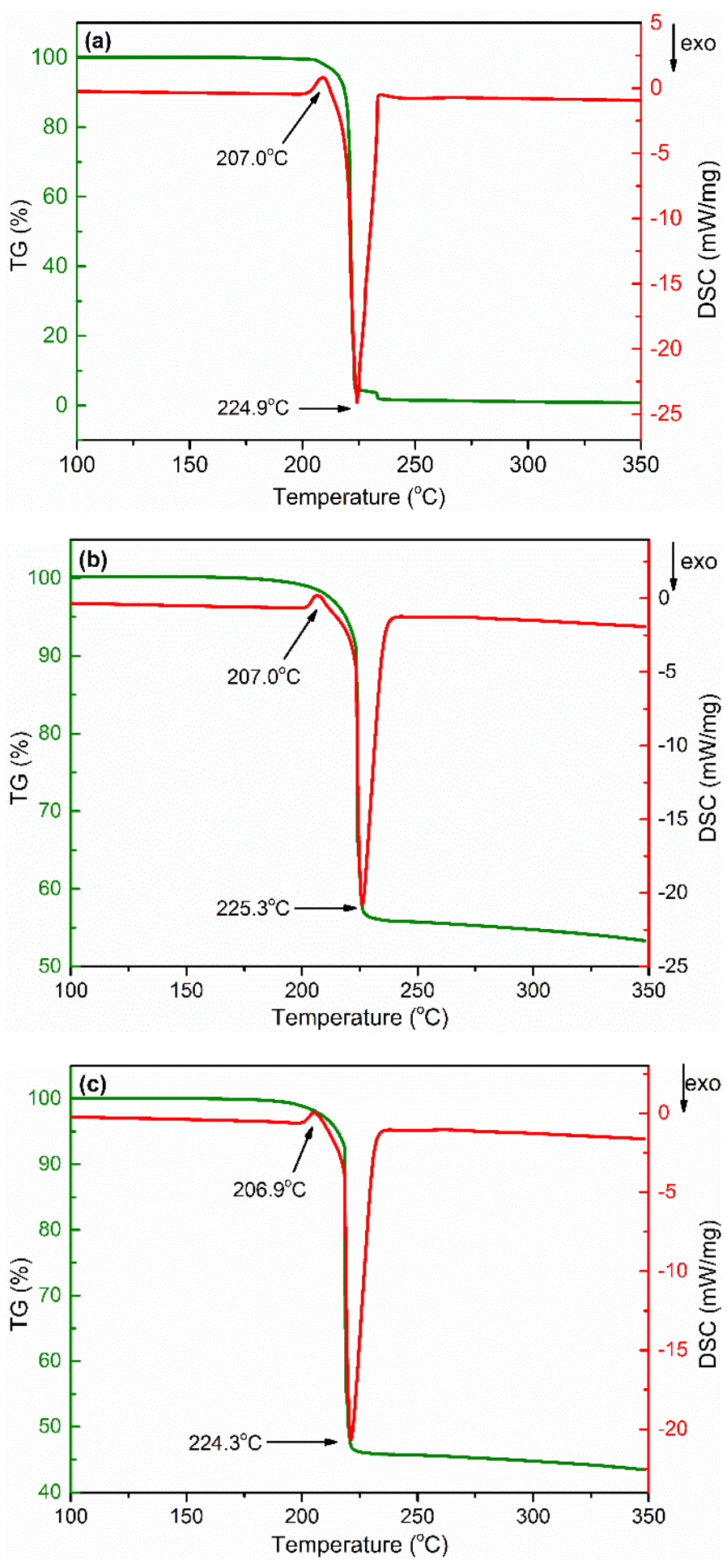

Figure S2 TG-DSC curves of (a) pureRDX, (b)HTPB/RDX mixture, (c) AEHTPB/HTPB/RDX mixture in nitrogen atm. 
Table S4 Burning rate and pressure exponent of propellants with different content of AEHTPB

\begin{tabular}{cccccc}
\hline \multirow{2}{*}{ AEHTPB content (wt.\%) } & \multicolumn{5}{c}{ Burning rate $(\mathrm{mm} / \mathrm{s})$} \\
\cline { 2 - 5 } & $3.5 \mathrm{MPa}$ & $5.5 \mathrm{MPa}$ & $7.5 \mathrm{MPa}$ & $9.5 \mathrm{MPa}$ & \\
\hline 0 & 5.53 & 6.71 & 7.69 & 8.54 & 0.436 \\
$1 \%$ & 5.45 & 6.65 & 0.76 & 8.44 & 0.436 \\
$2 \%$ & 5.35 & 6.47 & 7.40 & 8.20 & 0.429 \\
$3 \%$ & 5.26 & 6.37 & 7.26 & 8.03 & 0.424 \\
$4 \%$ & 5.09 & 6.16 & 7.00 & 7.74 & 0.418 \\
$5 \%$ & 5.03 & 6.06 & 6.90 & 7.62 & 0.415 \\
$6 \%$ & 5.02 & 6.07 & 6.90 & 7.61 & 0.414 \\
$7 \%$ & 5.02 & 6.05 & 6.89 & 7.59 & 0.413 \\
$8 \%$ & 5.01 & 6.04 & 6.87 & 7.58 & 0.414 \\
$9 \%$ & 5.00 & 6.03 & 6.86 & 7.56 & 0.413 \\
$10 \%$ & 4.99 & 6.02 & 6.84 & 7.55 & 0.413 \\
\hline
\end{tabular}

a: AEHTPB content refers to the weight percentage of AEHTPB in the binary mixture of AEHTPB/HTPB. 